\title{
PERIODIC SOLUTIONS OF A KIND OF LIÉNARD EQUATIONS WITH TWO DEVIATING ARGUMENTS
}

\author{
Tiantian Ma
}

\begin{abstract}
In this paper, we deal with the existence of periodic solutions of a kind of Liénard equations with two deviating arguments

$$
x^{\prime \prime}+f(t, x(t-\sigma(t))) x^{\prime}(t)+g(t, x(t-\tau(t)))=p(t) .
$$
\end{abstract}

Some new results on the existence of periodic solutions of the given equations are proved by using the continuation theorem.

\section{Introduction}

In recent years, the periodic problem of Liénard equations with deviating arguments has been widely studied because of its background in applied sciences (see [2], [5], [7], [9]-[13] and the references cited therein). In [7], Huang and Xiang studied the periodic solutions of Duffing equations with a single constant deviating argument

$$
x^{\prime \prime}+g(x(t-\tau))=p(t) .
$$

2010 Mathematics Subject Classification. 34K10, 34K13.

Key words and phrases. Liénard equation, deviating argument, periodic solution, continuation theorem.

Research supported by Research Fund for the Doctoral Program of Higher Education of China, No. 11AA0013, Beijing Natural Science Foundation (Existence and multiplicity of periodic solutions in nonlinear oscillations), No. 1112006 and the Grant of Beijing Education Committee Key Project, No. KZ201310028031. 
When $g$ satisfies one sign condition $x g(x)>0,|x|>M$ and a one-sided boundedness condition, the authors proved the existence of periodic solutions of equation (1.1). In [9], Liu and Huang studied the periodic solutions of Liénard equations with one deviating argument

$$
x^{\prime \prime}+f(x(t)) x^{\prime}(t)+g(t, x(t-\tau(t)))=p(t) .
$$

When $f, g$ are Lipschitz continuous, $f$ is bounded and $g$ satisfies one sign condition $x(g(t, x)-p(t))>0$ (or $<0)$ for $t \in \mathbb{R},|x| \geq d$, they obtained the existence and uniqueness of periodic solution of equation (1.2).

In [11], Lu and Ge discussed the existence of periodic solutions of Liénard equations with two deviating arguments

$$
x^{\prime \prime}+f(t, x(t-\sigma(t))) x^{\prime}(t)+\beta(t) g(x(t-\tau(t)))=p(t),
$$

where $f: \mathbb{R}^{2} \rightarrow \mathbb{R}$ is continuous and $2 \pi$ periodic with respect to the first variable, $g: \mathbb{R} \rightarrow \mathbb{R}$ is continuous and $\sigma, \beta, \tau, p: \mathbb{R} \rightarrow \mathbb{R}$ are continuous and $2 \pi$ periodic.

Assume that the following conditions are satisfied:

$$
\begin{gathered}
\lim _{|x| \rightarrow+\infty} \operatorname{sgn}(x) g(x)=+\infty, \\
\limsup _{|x| \rightarrow+\infty}\left|\frac{g(x)}{x}\right| \leq r .
\end{gathered}
$$

Moreover,

$$
0<\beta_{0}=\min _{t \in[0,2 \pi]} \beta(t) \leq \beta_{1}=\max _{t \in[0,2 \pi]} \beta(t) .
$$

When the conditions (1.4), (1.5) and (1.6) hold, it was proved in [11] that, if $|f(t, x)| \leq \varrho$ ( $\varrho$ is a positive constant) for $(t, x) \in \mathbb{R}^{2}$, then equation (1.3) has at least one $2 \pi$ periodic solution provided that the inequality $2 \pi\left(\varrho+2 \pi r \beta_{1}\right)<1$ holds and if $|f(t, x)| \geq \varrho$ for $(t, x) \in \mathbb{R}^{2}$, then equation (1.3) has at least one $2 \pi$ periodic solution provided that the inequality $2 \pi r \beta_{1}<\varrho$ holds.

In this paper, we deal with the existence of periodic solutions of a kind of Liénard equations with two deviating arguments

$$
x^{\prime \prime}+f(t, x(t-\sigma(t))) x^{\prime}(t)+g(t, x(t-\tau(t)))=p(t),
$$

where $f, g: \mathbb{R}^{2} \rightarrow \mathbb{R}$ are continuous and $2 \pi$ periodic with respect to the first variable, $\sigma, \tau, p: \mathbb{R} \rightarrow \mathbb{R}$ are continuous and $2 \pi$ periodic. By taking a new estimating method, we give some new conditions to guarantee the existence of periodic solutions of equation (1.7). Main results of this paper are the following theorems.

ThEOREM 1.1. Assume that there exist constants $\varrho \geq 0, d>0, r \geq 0$ and $c>0$ such that the following conditions hold:

$\left(\mathrm{f}_{1}\right)|f(t, x)| \leq \varrho$ for $(t, x) \in \mathbb{R}^{2} ;$ 
$\left(\mathrm{g}_{1}\right) \operatorname{sgn}(x) g(t, x)>l$ for $t \in \mathbb{R},|x|>d$, where $l=\max \{|p(t)|: t \in[0,2 \pi]\}$;

$\left(\mathrm{g}_{2}\right) g(t, x) \leq r x+c$ for $t \in \mathbb{R},|x| \geq d$.

Then equation (1.7) has at least one $2 \pi$ periodic solution provided that the inequality $2 \pi(\varrho+\pi r)<1$ holds.

Theorem 1.2. Assume that there exist constants $\varrho \geq 0, d>0, r \geq 0$ and $c>0$ such that the following conditions hold:

$\left(\mathrm{f}_{1}\right)|f(t, x)| \leq \varrho$ for $(t, x) \in \mathbb{R}^{2}$;

$\left(\mathrm{g}_{1}\right) \operatorname{sgn}(x) g(t, x)>l$ for $t \in \mathbb{R},|x|>d$, where $l=\max \{|p(t)|: t \in[0,2 \pi]\}$;

$\left(\mathrm{g}_{3}\right)|g(t, x)| \leq r|x|+c$ for $t \in \mathbb{R},|x| \geq d$.

Then equation (1.7) has at least one $2 \pi$ periodic solution provided that the inequality $\varrho+\pi r<1$ holds.

TheOREM 1.3. Assume that there exist constants $\varrho>0, d>0, r \geq 0$ and $c>0$ such that the following conditions hold:

$\left(\mathrm{f}_{2}\right)|f(t, x)| \geq \varrho$ for $(t, x) \in \mathbb{R}^{2}$;

$\left(\mathrm{g}_{1}\right) \operatorname{sgn}(x) g(t, x)>l$ for $t \in \mathbb{R},|x|>d$, where $l=\max \{|p(t)|: t \in[0,2 \pi]\}$;

$\left(\mathrm{g}_{3}\right)|g(t, x)| \leq r|x|+c$ for $t \in \mathbb{R},|x| \geq d$.

Then equation (1.7) has at least one $2 \pi$ periodic solution provided that the inequality $\pi r<\varrho$ holds.

REMARK 1.4. The main Theorems 1.1-1.3 improve and complement the results in [11]. In fact, if we take $g(t, x)=\beta(t) g(x)$, then we have

$$
\limsup _{|x| \rightarrow+\infty}\left|\frac{g(t, x)}{x}\right| \leq \beta_{1} r
$$

provided that the conditions (1.5) and (1.6) hold. When the conditions (1.4)(1.6) hold, we know from Theorem 1.2 that, if $|f(t, x)| \leq \varrho$ ( $\varrho$ is a positive constant) for $(t, x) \in \mathbb{R}^{2}$, then equation (1.3) has at least one $2 \pi$ periodic solution provided that the inequality $\varrho+\pi r \beta_{1}<1$ holds and we know from Theorem 2.3 that, if $|f(t, x)| \geq \varrho$ for $(t, x) \in \mathbb{R}^{2}$, then equation (1.3) has at least one $2 \pi$ periodic solution provided that the inequality $\pi r \beta_{1}<\varrho$ holds. Therefore, Theorems 1.2 and 1.3 improve the results in [11].

Throughout this paper, for any continuous $2 \pi$ periodic function $x(t)$, we always use notations as follows,

$$
\|x\|_{\infty}=\max _{t \in[0,2 \pi]}\{|x(t)|\}, \quad\|x\|_{2}=\left(\int_{0}^{2 \pi} x^{2}(t) d t\right)^{1 / 2} .
$$

\section{Preliminary lemmas}

As is well known that continuation theorems play an important role in studying the existence of periodic solutions of the second order differential equations. We now introduce a continuation theorem given by Gaines and Mawhin ([4]). 
Lemma 2.1. Let $X$ and $Y$ be two Banach spaces. Suppose that $L: D \subset X \rightarrow Y$ is a Fredholm operator with index zero and $N: X \rightarrow Y$ is L-compact on $\bar{\Omega}$, where $\Omega$ is an open bounded subset of $X$. Moreover, assume that all the following conditions are satisfied:

(a) $L x \neq \lambda N x$, for all $x \in \partial \Omega \cap D(L), \lambda \in(0,1)$;

(b) $N x \notin \operatorname{Im} L$, for all $x \in \partial \Omega \cap \operatorname{Ker} L$;

(c) The Brower degree $\operatorname{deg}\{Q N, \Omega \cap \operatorname{Ker} L, 0\} \neq 0$.

Then equation $L x=N x$ has at least one solution in $\bar{\Omega}$.

Next, we shall give two lemmas which will be used in getting the prior bounds.

Lemma 2.2. Let $x(t)$ be a continuously differentiable $2 \pi$ periodic function. Then, for any $t \in[0,2 \pi]$,

$$
\|x\|_{\infty} \leq|x(t)|+\pi\left\|x^{\prime}\right\|_{\infty}
$$

Proof. Let $\bar{t} \in[t, t+2 \pi]$ be such that $|x(\bar{t})|=\|x\|_{\infty}$. Then we have

$$
|x(\bar{t})|=\left|x(t)+\int_{t}^{\bar{t}} x^{\prime}(s) d s\right| \leq|x(t)|+\int_{t}^{\bar{t}}\left|x^{\prime}(s)\right| d s .
$$

On the other hand, we have

$$
|x(\bar{t})|=|x(\bar{t}-2 \pi)|=\left|x(t)+\int_{t}^{\bar{t}-2 \pi} x^{\prime}(s) d s\right| \leq|x(t)|+\int_{\bar{t}-2 \pi}^{t}\left|x^{\prime}(s)\right| d s .
$$

Therefore,

$$
\|x\|_{\infty} \leq|x(t)|+\frac{1}{2} \int_{0}^{2 \pi}\left|x^{\prime}(t)\right| d t
$$

which implies

$$
\|x\|_{\infty} \leq|x(t)|+\pi\left\|x^{\prime}\right\|_{\infty}
$$

Lemma 2.3. Let $x(t)$ be a twice continuously differentiable $2 \pi$ periodic function. Then

$$
\int_{0}^{2 \pi}\left|x^{\prime}(t)\right|^{2} d t \leq \int_{0}^{2 \pi}\left|x^{\prime \prime}(t)\right|^{2} d t
$$

PROOF. The proof follows directly from the Wirtinger inequality ([6]).

\section{Main theorems}

In this section, we shall use the continuation theorem introduced in Section 2 to prove the existence of periodic solutions of equation (1.7). To this end, we first quote some notations and definitions.

Let $X$ and $Y$ be two Banach spaces defined by

$$
\begin{aligned}
& X=\left\{x \in C^{1}(\mathbb{R}, \mathbb{R}): x(t+2 \pi)=x(t), \text { for all } t \in \mathbb{R}\right\}, \\
& Y=\{y \in C(\mathbb{R}, \mathbb{R}): y(t+2 \pi)=y(t), \text { for all } t \in \mathbb{R}\}
\end{aligned}
$$


with the norms

$$
\|x\|_{X}=\max \left\{\|x\|_{\infty},\left\|x^{\prime}\right\|_{\infty}\right\}, \quad\|y\|_{Y}=\|y\|_{\infty} .
$$

Define a linear operator

$$
L: D(L) \subset X \rightarrow Y, \quad L x=x^{\prime \prime},
$$

where $D(L)=\left\{x \in X: x^{\prime \prime} \in C(\mathbb{R}, \mathbb{R})\right\}$, and a nonlinear operator

$$
N: X \rightarrow Y, \quad(N x)(t)=-f(t, x(t-\sigma(t))) x^{\prime}(t)-g(t, x(t-\tau(t)))+p(t) .
$$

It is easy to see that

$$
\operatorname{Ker} L=\mathbb{R} \text { and } \operatorname{Im} L=\left\{y \in Y: \int_{0}^{2 \pi} y(t) d t=0\right\} .
$$

It follows that $L$ is a Fredholm mapping of index zero.

Let us define two continuous projectors $P: X \rightarrow \operatorname{Ker} L$ and $Q: Y \rightarrow Y$ by setting

$$
P x=x(0), \quad Q y=\frac{1}{2 \pi} \int_{0}^{2 \pi} y(t) d t .
$$

Set $L_{P}=\left.L\right|_{D(L) \cap \operatorname{Ker} P} \rightarrow \operatorname{Im} L$. Then $L_{P}$ is an algebraic isomorphism and we define $K_{P}: \operatorname{Im} L \rightarrow D(L)$ by

$$
K_{P}=L_{P}^{-1}
$$

Clearly, we have that, for any $y \in \operatorname{Im} L$,

$$
\left(K_{P} y\right)(t)=-\frac{t}{2 \pi} \int_{0}^{2 \pi}(t-s) y(s) d s+\int_{0}^{t}(t-s) y(s) d s .
$$

For any open bounded set $\Omega \subset X$, we can prove by standard arguments that $K_{P}(I-Q) N$ and $Q N$ are relatively compact on the closure $\bar{\Omega}$. Therefore, $N$ is $L$-compact on $\bar{\Omega}$.

It is noted that equation (1.7) is equivalent to the operator equation

$$
L x=N x .
$$

To use Lemma 2.1, we embed this operator equation into an equation family with a parameter $\lambda \in(0,1)$,

$$
L x=\lambda N x,
$$

which is equivalent to the equation as follows,

$$
x^{\prime \prime}+\lambda f(t, x(t-\sigma(t))) x^{\prime}(t)+\lambda g(t, x(t-\tau(t)))=\lambda p(t), \quad \lambda \in(0,1) .
$$

In the following, we shall prove some new theorems on the existence of periodic solutions of equation (1.7) by using the continuation theorem. By standard arguments [4], it suffices to prove that there exist positive constants $M_{1}$ and $M_{2}$ such that, for any $2 \pi$ periodic solution $x(t)$ of equation (3.1),

$$
\|x\|_{\infty}<M_{1}, \quad\left\|x^{\prime}\right\|_{\infty}<M_{2} .
$$


THEOREM 3.1. Assume that there exist constants $\varrho \geq 0, d>0, r \geq 0$ and $c>0$ such that the following conditions hold:

$\left(\mathrm{f}_{1}\right)|f(t, x)| \leq \varrho$ for $(t, x) \in \mathbb{R}^{2}$

$\left(\mathrm{g}_{1}\right) \operatorname{sgn}(x) g(t, x)>l$ for $t \in \mathbb{R},|x|>d$, where $l=\max \{|p(t)|: t \in[0,2 \pi]\}$;

$\left(\mathrm{g}_{2}\right) g(t, x) \leq r x+c$ for $t \in \mathbb{R}, x \geq d$.

Then equation (1.7) has at least one $2 \pi$ periodic solution provided that the inequality $2 \pi(\varrho+\pi r)<1$ holds.

Proof. We shall prove that all conditions of Lemma 2.1 are satisfied. Consider the auxiliary equation (3.1). Let $x(t)$ be any $2 \pi$ periodic solution of equation (3.1). Then there exist $t_{*}, t^{*} \in[0,2 \pi]$ such that

$$
x\left(t_{*}\right)=\min _{t \in[0,2 \pi]} x(t), \quad x\left(t^{*}\right)=\max _{t \in[0,2 \pi]} x(t) .
$$

It follows that $x^{\prime}\left(t_{*}\right)=0, x^{\prime \prime}\left(t_{*}\right) \geq 0 ; x^{\prime}\left(t^{*}\right)=0, x^{\prime \prime}\left(t^{*}\right) \leq 0$. Therefore,

$$
g\left(t_{*}, x\left(t_{*}-\tau\left(t_{*}\right)\right)\right) \leq l, \quad g\left(t^{*}, x\left(t^{*}-\tau\left(t^{*}\right)\right)\right) \geq-l .
$$

According to $\left(g_{1}\right)$, we get

$$
x\left(t_{*}-\tau\left(t_{*}\right)\right) \leq d, \quad x\left(t^{*}-\tau\left(t^{*}\right)\right) \geq-d .
$$

Consequently, there exists $\bar{t} \in[0,2 \pi]$ such that $|x(\bar{t}-\tau(\bar{t}))| \leq d$. Since $x(t)$ and $\tau(t)$ are $2 \pi$ periodic, there exists $\tilde{t} \in[0,2 \pi]$ such that

$$
|x(\widetilde{t})| \leq d
$$

From Lemma 2.2 and (3.2) we have

$$
\|x\|_{\infty} \leq d+\pi\left\|x^{\prime}\right\|_{\infty} .
$$

Write $S_{1}=\{t \in[0,2 \pi]: x(t-\tau(t))>d\}, S_{2}=\{t \in[0,2 \pi]: x(t-\tau(t))<-d\}$, $S_{3}=\{t \in[0,2 \pi]:|x(t-\tau(t))| \leq d\}$. Since

$$
\int_{0}^{2 \pi} f(t, x(t-\sigma(t))) x^{\prime}(t) d t+\int_{0}^{2 \pi} g(t, x(t-\tau(t))) d t=\int_{0}^{2 \pi} p(t) d t
$$

we get

$$
\begin{aligned}
-\int_{S_{2}} g(t, x(t-\tau(t))) d t= & \int_{0}^{2 \pi} f(t, x(t-\sigma(t))) x^{\prime}(t) d t \\
& +\left(\int_{S_{1}}+\int_{S_{3}}\right) g(t, x(t-\tau(t))) d t-\int_{0}^{2 \pi} p(t) d t .
\end{aligned}
$$

Furthermore,

$$
\begin{gathered}
\int_{S_{2}}|g(t, x(t-\tau(t)))| d t=-\int_{S_{2}} g(t, x(t-\tau(t))) d t \\
\leq \int_{0}^{2 \pi}\left|f(t, x(t-\sigma(t))) x^{\prime}(t)\right| d t
\end{gathered}
$$




$$
+\left(\int_{S_{1}}+\int_{S_{3}}\right)|g(t, x(t-\tau(t)))| d t+\int_{0}^{2 \pi}|p(t)| d t .
$$

From (2.1) and (3.4) we have

$$
\begin{aligned}
\left\|x^{\prime}\right\|_{\infty} \leq \frac{1}{2} \int_{0}^{2 \pi}\left|x^{\prime \prime}(t)\right| d t \leq & \frac{1}{2} \int_{0}^{2 \pi}\left|f(t, x(t-\sigma(t))) x^{\prime}(t)\right| d t \\
& +\frac{1}{2} \int_{0}^{2 \pi}|g(t, x(t-\tau(t)))| d t+\frac{1}{2} \int_{0}^{2 \pi}|p(t)| d t \\
\leq & \int_{0}^{2 \pi}\left|f(t, x(t-\sigma(t))) x^{\prime}(t)\right| d t \\
& +\left(\int_{S_{1}}+\int_{S_{3}}\right)|g(t, x(t-\tau(t)))| d t+\int_{0}^{2 \pi}|p(t)| d t .
\end{aligned}
$$

Furthermore, from $\left(\mathrm{f}_{1}\right),\left(\mathrm{g}_{2}\right)$ and (3.3) that

$$
\begin{aligned}
\left\|x^{\prime}\right\|_{\infty} & \leq 2 \varrho \pi\left\|x^{\prime}\right\|_{\infty}+2 r \pi\|x\|_{\infty}+2 \pi\left(c+M_{d}+l\right) \\
& \leq 2 \varrho \pi\left\|x^{\prime}\right\|_{\infty}+2 r \pi\left(d+\pi\left\|x^{\prime}\right\|_{\infty}\right)+2 \pi\left(c+M_{d}+l\right) \\
& =2 \pi(\varrho+r \pi)\left\|x^{\prime}\right\|_{\infty}+2 \pi\left(r d+M_{d}+c+l\right),
\end{aligned}
$$

where $M_{d}=\max \{|g(x)|:|x| \leq d\}$.

Since $2 \pi(\varrho+r \pi)<1$, there exists a constant $M_{1}>0$ such that $\left\|x^{\prime}\right\|_{\infty} \leq M_{1}$. According to (3.3), we obtain $\|x\|_{\infty} \leq d+\pi M_{1}$.

Similarly, we have the following result.

THEOREM 3.1'. Assume that there exist constants $\varrho \geq 0, d>0, r \geq 0$ and $c>0$ such that the following conditions hold:

$\left(\mathrm{f}_{1}\right)|f(t, x)| \leq \varrho$ for $(t, x) \in \mathbb{R}^{2}$;

$\left(\mathrm{g}_{1}\right) \operatorname{sgn}(x) g(t, x)>l$ for $t \in \mathbb{R},|x|>d$, where $l=\max \{|p(t)|: t \in[0,2 \pi]\}$;

$\left(\mathrm{g}_{2}^{\prime}\right) g(t, x) \geq r x-c$ for $t \in \mathbb{R}, x \leq-d$.

Then equation (1.7) has at least one $2 \pi$ periodic solution provided that the inequality $2 \pi(\varrho+\pi r)<1$ holds.

REMARK 3.2. The conclusion of Theorem 3.1 (or Theorem 3.1') still holds if the condition $\left(\mathrm{g}_{1}\right)$ is replaced by the condition:

$\left(\mathrm{g}_{1}^{\prime}\right) \operatorname{sgn}(x) g(t, x)<-l$ for $t \in[0,2 \pi],|x|>d$, where $l=\max \{|p(t)|: t \in$ $[0,2 \pi]\}$,

whereas condition $\left(\mathrm{g}_{2}\right)$ (or $\left.\left(\mathrm{g}_{2}^{\prime}\right)\right)$ is replaced by the condition:

$\left(\mathrm{g}_{2}^{\prime \prime}\right) g(t, x) \geq-r x-c$ for $t \in \mathbb{R}, x \geq d,($ or $g(t, x) \leq-r x+c$ for $t \in \mathbb{R}$, $x \leq-d)$.

Corollary 3.3. Assume that conditions $\left(\mathrm{f}_{1}\right)$ and $\left(\mathrm{g}_{1}\right)$ hold. Moreover, $g$ satisfies the sublinear condition as follows:

$$
\lim _{x \rightarrow+\infty} \frac{g(t, x)}{x}=0 \quad \text { or } \quad \lim _{x \rightarrow-\infty} \frac{g(t, x)}{x}=0
$$


uniformly for $t \in[0,2 \pi]$. Then equation (1.7) has at least one $2 \pi$ periodic solution provided that the inequality $2 \pi \varrho<1$ holds.

Proof. Since $2 \pi \varrho<1$, there exists a constant $\varepsilon>0$ such that $2 \pi(\varrho+\varepsilon \pi)<1$. From (3.5) we know that there exists a constant $d_{\varepsilon}>0$ such that

$$
g(t, x) \leq \varepsilon x \quad \text { for } t \in[0,2 \pi], x \geq d_{\varepsilon}
$$

or

$$
g(t, x) \geq \varepsilon x \quad \text { for } t \in[0,2 \pi], x \leq-d_{\varepsilon} .
$$

If (3.6) holds, then we know from Theorem 3.1 that equation (1.7) has at least one $2 \pi$ periodic solution. If (3.7) holds, then we know from Theorem $3.1^{\prime}$ that equation (1.7) has at least one $2 \pi$ periodic solution.

In the following, we shall deal with the existence of periodic solutions of equation (1.7) when the condition $\left(\mathrm{g}_{2}\right)$ ( or $\left(\mathrm{g}_{2}^{\prime}\right)$ ) is replaced by one double sided condition.

THEOREM 3.4. Assume that there exist constants $\varrho \geq 0, d>0, r \geq 0$ and $c>0$ such that the following conditions hold:

$\left(\mathrm{f}_{1}\right)|f(t, x)| \leq \varrho$ for $(t, x) \in \mathbb{R}^{2}$

$\left(\mathrm{g}_{1}\right) \operatorname{sgn}(x) g(t, x)>l$ for $t \in \mathbb{R},|x|>d$, where $l=\max \{|p(t)|: t \in[0,2 \pi]\}$;

$\left(\mathrm{g}_{3}\right)|g(t, x)| \leq r|x|+c$ for $t \in \mathbb{R},|x| \geq d$.

Then equation (1.7) has at least one $2 \pi$ periodic solution provided that the inequality $\varrho+\pi r<1$ holds.

Proof. Consider the auxiliary equation (3.1). Let $x(t)$ be any $2 \pi$ periodic solution of equation (3.1). Multiplying both sides of (3.1) by $x^{\prime \prime}(t)$ and integrating on the interval $[0,2 \pi]$, we have

$$
\begin{aligned}
\int_{0}^{2 \pi} x^{\prime \prime}(t)^{2} d t= & -\lambda \int_{0}^{2 \pi} f(t, x(t-\sigma(t))) x^{\prime}(t) x^{\prime \prime}(t) d t \\
& -\lambda \int_{0}^{2 \pi} g(t, x(t-\tau(t))) x^{\prime \prime}(t) d t+\lambda \int_{0}^{2 \pi} p(t) x^{\prime \prime}(t) d t \\
\leq & \int_{0}^{2 \pi}\left|f(t, x(t-\sigma(t))) x^{\prime}(t) x^{\prime \prime}(t)\right| d t \\
& +\int_{0}^{2 \pi}\left|g(t, x(t-\tau(t))) x^{\prime \prime}(t)\right| d t+\int_{0}^{2 \pi}\left|p(t) x^{\prime \prime}(t)\right| d t .
\end{aligned}
$$

According to the conditions $\left(\mathrm{f}_{1}\right)$ and $\left(\mathrm{g}_{3}\right)$, we get

$$
\begin{aligned}
\int_{0}^{2 \pi} x^{\prime \prime}(t)^{2} d t \leq & \varrho \int_{0}^{2 \pi}\left|x^{\prime}(t) x^{\prime \prime}(t)\right| d t \\
& +r \int_{0}^{2 \pi}\left|x(t-\tau(t)) x^{\prime \prime}(t)\right| d t+(c+l) \int_{0}^{2 \pi}\left|x^{\prime \prime}(t)\right| d t
\end{aligned}
$$




$$
\begin{aligned}
\leq & \varrho\left(\int_{0}^{2 \pi} x^{\prime}(t)^{2} d t\right)^{1 / 2}\left(\int_{0}^{2 \pi} x^{\prime \prime}(t)^{2} d t\right)^{1 / 2} \\
& +r\|x\|_{\infty} \int_{0}^{2 \pi}\left|x^{\prime \prime}(t)\right| d t+(c+l) \int_{0}^{2 \pi}\left|x^{\prime \prime}(t)\right| d t .
\end{aligned}
$$

From (2.1), (3.2) and Lemma 2.3, we have

$$
\begin{aligned}
\int_{0}^{2 \pi} x^{\prime \prime}(t)^{2} d t \leq & \varrho \int_{0}^{2 \pi} x^{\prime \prime}(t)^{2} d t \\
& +r\left(d+\frac{1}{2} \int_{0}^{2 \pi}\left|x^{\prime}(t)\right| d t\right) \int_{0}^{2 \pi}\left|x^{\prime \prime}(t)\right| d t+(c+l) \int_{0}^{2 \pi}\left|x^{\prime \prime}(t)\right| d t \\
\leq & \varrho \int_{0}^{2 \pi} x^{\prime \prime}(t)^{2} d t+r \pi\left(\int_{0}^{2 \pi} x^{\prime}(t)^{2} d t\right)^{1 / 2}\left(\int_{0}^{2 \pi} x^{\prime \prime}(t)^{2} d t\right)^{1 / 2} \\
& +(r d+c+l) \int_{0}^{2 \pi}\left|x^{\prime \prime}(t)\right| d t \\
\leq & (\varrho+r \pi) \int_{0}^{2 \pi} x^{\prime \prime}(t)^{2} d t+(r d+c+l) \sqrt{2 \pi}\left(\int_{0}^{2 \pi} x^{\prime \prime}(t)^{2} d t\right)^{1 / 2} .
\end{aligned}
$$

Since $\varrho+r \pi<1$, there exists a constant $M_{1}>0$ such that $\left\|x^{\prime \prime}\right\|_{2} \leq M_{1}$. Furthermore, we have

$$
\left\|x^{\prime}\right\|_{\infty} \leq \frac{1}{2} \int_{0}^{2 \pi}\left|x^{\prime \prime}(t)\right| d t \leq \frac{\sqrt{2 \pi} M_{1}}{2}=: M_{2} .
$$

From (3.8) and Lemma 2.2 we get $\|x\|_{\infty} \leq d+\left\|x^{\prime}\right\|_{\infty} \leq d+M_{2}$.

Corollary 3.5. Assume that conditions $\left(\mathrm{f}_{1}\right)$ and $\left(\mathrm{g}_{1}\right)$ hold. Moreover, $g$ satisfies the sublinear condition as follows:

$$
\lim _{|x| \rightarrow+\infty} \frac{g(t, x)}{x}=0
$$

uniformly for $t \in[0,2 \pi]$. Then equation (1.7) has at least one $2 \pi$ periodic solution provided that the inequality $\varrho<1$ holds.

Proof. Since $\varrho<1$, there exists a constant $\varepsilon>0$ such that $\varrho+\varepsilon \pi<1$. If (3.9) holds, then there exists a constant $d_{\varepsilon}>0$ such that $|g(t, x)| \leq \varepsilon|x|$ for $t \in[0,2 \pi],|x| \geq d_{\varepsilon}$. According to Theorem 3.4, equation (1.7) has at least one $2 \pi$ periodic solution.

In the case when $f$ is unbounded, we can obtain the following result.

Theorem 3.6. Assume that there exist constants $\varrho>0, d>0, r \geq 0$ and $c>0$ such that the following conditions hold:

$\left(\mathrm{f}_{2}\right)|f(t, x)| \geq \varrho$ for $(t, x) \in \mathbb{R}^{2}$;

$\left(\mathrm{g}_{1}\right) \operatorname{sgn}(x) g(t, x)>l$ for $t \in \mathbb{R},|x|>d$, where $l=\max \{|p(t)|: t \in[0,2 \pi]\}$;

$\left(\mathrm{g}_{3}\right)|g(t, x)| \leq r|x|+c$ for $t \in \mathbb{R},|x| \geq d$. 
Then equation (1.7) has at least one $2 \pi$ periodic solution provided that the inequality $\pi r<\varrho$ holds.

Proof. We only deal with the case $f(t, x) \geq \varrho$ for $(t, x) \in \mathbb{R}^{2}$. Another case $f(t, x) \leq-\varrho$ for $(t, x) \in \mathbb{R}^{2}$ can be handled similarly. Consider the auxiliary equation (3.1). Let $x(t)$ be any $2 \pi$ periodic solution of equation (3.1). Multiplying both sides of $(3.1)$ by $x^{\prime}(t)$ and integrating on the interval $[0,2 \pi]$, we get

$$
\int_{0}^{2 \pi} f(t, x(t-\sigma(t))) x^{\prime}(t)^{2} d t+\int_{0}^{2 \pi} g(t, x(t-\tau(t))) x^{\prime}(t) d t=\int_{0}^{2 \pi} p(t) x^{\prime}(t) d t .
$$

According to $\left(\mathrm{f}_{2}\right)$, we have

$$
\begin{aligned}
\varrho \int_{0}^{2 \pi} x^{\prime}(t)^{2} d t & \leq \int_{0}^{2 \pi} f(t, x(t-\sigma(t))) x^{\prime}(t)^{2} d t \\
& =-\int_{0}^{2 \pi} g(t, x(t-\tau(t))) x^{\prime}(t) d t+\int_{0}^{2 \pi} p(t) x^{\prime}(t) d t .
\end{aligned}
$$

Hence,

$$
\varrho \int_{0}^{2 \pi} x^{\prime}(t)^{2} d t \leq \int_{0}^{2 \pi}|g(t, x(t-\tau(t)))|\left|x^{\prime}(t)\right| d t+\int_{0}^{2 \pi}|p(t)|\left|x^{\prime}(t)\right| d t .
$$

From condition $\left(\mathrm{g}_{3}\right)$ we obtain

$$
\varrho \int_{0}^{2 \pi} x^{\prime}(t)^{2} d t \leq r\|x\|_{\infty} \int_{0}^{2 \pi}\left|x^{\prime}(t)\right| d t+(l+c) \int_{0}^{2 \pi}\left|x^{\prime}(t)\right| d t
$$

which, together with (2.1) and (3.2), implies

$$
\begin{aligned}
\varrho \int_{0}^{2 \pi} x^{\prime}(t)^{2} d t & \leq r\left(d+\frac{1}{2} \int_{0}^{2 \pi}\left|x^{\prime}(t)\right| d t\right) \int_{0}^{2 \pi}\left|x^{\prime}(t)\right| d t+(l+c) \int_{0}^{2 \pi}\left|x^{\prime}(t)\right| d t \\
& \leq r \pi \int_{0}^{2 \pi} x^{\prime}(t)^{2} d t+(r d+l+c) \sqrt{2 \pi}\left(\int_{0}^{2 \pi} x^{\prime}(t)^{2} d t\right)^{1 / 2} .
\end{aligned}
$$

Since $r \pi<\varrho$, there exists a constant $M_{1}>0$ such that

$$
\left\|x^{\prime}\right\|_{2} \leq M_{1} \text {. }
$$

Furthermore, we get

$\|x\|_{\infty} \leq d+\frac{1}{2} \int_{0}^{2 \pi}\left|x^{\prime}(t)\right| d t \leq d+\frac{\sqrt{2 \pi}}{2}\left(\int_{0}^{2 \pi} x^{\prime}(t)^{2} d t\right)^{1 / 2} \leq d+\frac{\sqrt{2 \pi}}{2} M_{1}=: M_{2}$.

On the other hand, we have

$$
\begin{aligned}
\left\|x^{\prime}\right\|_{\infty} \leq \frac{1}{2} \int_{0}^{2 \pi}\left|x^{\prime \prime}(t)\right| & d t \leq \frac{1}{2}\left(\int_{0}^{2 \pi}\left|f(t, x(t-\sigma(t))) \| x^{\prime}(t)\right| d t\right. \\
+ & \left.\int_{0}^{2 \pi}|g(t, x(t-\tau(t)))| d t\right)+\frac{1}{2} \int_{0}^{2 \pi}|p(t)| d t .
\end{aligned}
$$


Set

$$
\begin{aligned}
& c_{1}=\max \left\{|f(t, x)|: 0 \leq t \leq 2 \pi,|x| \leq M_{2}\right\}, \\
& c_{2}=\max \left\{|g(t, x)|: 0 \leq t \leq 2 \pi,|x| \leq M_{2}\right\} .
\end{aligned}
$$

From (3.10)-(3.13) we obtain

$$
\left\|x^{\prime}\right\|_{\infty} \leq \frac{1}{2} c_{1} \int_{0}^{2 \pi}\left|x^{\prime}(t)\right| d t+\left(c_{2}+l\right) \pi \leq \frac{\sqrt{2 \pi}}{2} c_{1} M_{1}+\left(c_{2}+l\right) \pi .
$$

REMARK 3.7. The conclusion of Theorem 3.4 (or Theorem 3.6) still holds if the condition $\left(\mathrm{g}_{1}\right)$ is replaced by the condition $\left(\mathrm{g}_{1}^{\prime}\right)$.

COROLlary 3.8. Assume that the conditions $\left(\mathrm{f}_{2}\right)$ and $\left(\mathrm{g}_{1}\right)$ hold. Moreover, $g$ satisfies the sublinear condition (3.9). Then equation (1.7) has at least one $2 \pi$ periodic solution.

Proof. Since $\varrho>0$, there exists a constant $\varepsilon>0$ such that $\varepsilon \pi<\varrho$. If (3.9) holds, then there exists a constant $d_{\varepsilon}>0$ such that $|g(t, x)| \leq \varepsilon|x|$, for $t \in[0,2 \pi]$ and $|x| \geq d_{\varepsilon}$. According to Theorem 3.6, equation (1.7) has at least one $2 \pi$ periodic solution.

From Corollary 3.8 we can derive the following result.

Corollary 3.9. Assume that the conditions $\left(\mathrm{f}_{2}\right),\left(\mathrm{g}_{1}\right)$ hold and $g$ is bounded on $\mathbb{R}$. Then equation (1.7) has at least one $2 \pi$ periodic solution.

\section{REFERENCES}

[1] P. Amster, P. De Napoli And M.C. Mariani, Periodic solutions for p-Laplacian like systems with delay, Dyn. Contin. Discrete Impuls. Syst. Ser. A Math. Anal. 13 (2006), $311-319$.

[2] T.A. Burton And B. Zhang, Boundedness, periodicity, and convergence of solutions in a retarded Liénard equation, Ann. Mat. Pura Appl. (4) (1993), 351-368.

[3] W. Cheung and J. Ren, Periodic solutions for p-Laplacian Liénard equation with a deviating argument, Nonlinear Anal. 59 (2004), 107-120.

[4] R.E. Gaines and J.L. Mawhin, Coincidence degree and nonlinear differential equations, Springer, Berlin, 1977.

[5] W. GE, On the existence of harmonic solution of Liénard systems, Nonlinear Anal. 16 (1991), 183-190.

[6] G.H. Hardy, J.E. Littlewood and G. Polya, Inequalities, Cambridge University Press, London, 1964.

[7] X. Huang And Z. XIAng, On the existence of $2 \pi$ periodic solutions of Duffing type equation $x^{\prime \prime}(t)+g(x(t-\tau))=p(t)$, Chinese Sci. Bull. 39 (1994), 201-203. (in Chinese)

[8] B. Liu, Periodic solutions for Liénard type p-Laplacian equation with a deviating argument, J. Comput. Appl. Math. 214 (2008), 13-18.

[9] B. LiU AND L. HUANG, Existence and uniqueness of periodic solutions for a kind of Liénard equation with a deviating argument, Appl. Math. Lett. 21 (2008), 56-62.

[10] S. LU AND W. GE, Periodic solutions for a kind of Liénard equation with a deviating argument, J. Math. Anal. Appl. 289 (2004), 231-243. 
[11] _ On the existence of periodic solutions of second order differential equations with deviating arguments, Acta. Math. Sinica 45 (2002), 811-818.

[12] S. MA, Z.C. WANG AND J. Yu, An abstract theorem at resonance and its applications, J. Differential Equations 145 (1998), 274-294.

[13] _ Coincidence degree and periodic solutions of Duffing equations, Nonlinear Anal. 34 (1998), 443-460.

[14] Q. Zhou AND F. LONG, Existence and uniqueness of periodic solutions for a kind of Liénard equation with two deviating arguments, J. Comput. Appl. Math. 206 (2007), $1127-1136$.

Tiantian MA

School of Mathematical Sciences

Capital Normal University

Beijing 100048, P.R. CHINA

E-mail address: matiantian1215@163.com 